\title{
LOCAL SPECTRAL PROPERTIES OF CONVOLUTION OPERATORS ON NON-ABELIAN GROUPS
}

\author{
by VOLKER RUNDE
}

(Received 14th July 1994)

\begin{abstract}
Let $G$ be a Moore group. Then, for each $f \in L^{1}(G)$, the convolution operator $L_{f}: L^{1}(G) \rightarrow L^{1}(G)$ is decomposable. On the other hand, there is a discrete probability measure $\mu$ on a compact group $G$ such that $L_{\mu}: L^{1}(G) \rightarrow L^{1}(G)$ fails to be decomposable.
\end{abstract}

1991 Mathematics Subject Classification: 43A22, 47A11.

Since its early days, local spectral theory has had close connections to harmonic analysis: For a locally compact abelian (LCA) group $G$ and $f \in L^{1}(G)$, the convolution operator $L_{f}: L^{1}(G) \rightarrow L^{1}(G), g \rightarrow f * g$ is decomposable ([5, Theorem 6.2.11]). In [5], I. Colojoară and C. Foiaş asked if, for every LCA group $G$ and for each $\mu \in M(G)$, the convolution operator $L_{\mu}: L^{1}(G) \rightarrow L^{1}(G)$ is decomposable as well. This question was settled in the negative, independently, by. E. Albrecht ([2]) and J. Eschmeier ([7]): for every non-discrete LCA group $G$, there is $\mu \in L^{1}(G)$ such that $L_{\mu}: L^{1}(G) \rightarrow L^{1}(G)$ fails to be decomposable. The problem, for a non-discrete LCA group $G$, to characterize those $\mu \in M(G)$ which yield decomposable convolution operators is still open (see [14] for partial results).

If $G$ is a compact group, an old result by C. A. Akemann ([1, Theorem 4]) asserts that for each $f \in L^{1}(G)$ the convolution operator $L_{f}: L^{1}(G) \rightarrow L^{1}(G)$ is compact and thus decomposable. This indicates that also outside the realm of LCA groups convolution operators may enjoy significant local spectral properties.

The problem we are concerned with in this note reads-broadly phrased-as follows:

Let $G$ be a locally compact group, and let $E$ be a Banach space on which $L^{1}(G)$ acts via convolution as bounded linear operators (e.g. $C_{0}(G), M(G), L^{p}(G)$ for $p \in[1, \infty]$, or $C^{*}(G)$ ). When are the convolution operators $L_{f}: E \rightarrow E$ decomposable for all $f \in L^{1}(G)$ ?

If $f \in L^{1}(G)$ is self-adjoint, then $L_{f}: L^{2}(G) \rightarrow L^{2}(G)$ is a self-adjoint, bounded linear operator on a Hilbert space and thus decomposable. However, already the demand that $L_{f}: L^{1}(G) \rightarrow L^{1}(G)$ be decomposable for each self-adjoint $f \in L^{1}(G)$ has striking consequences for the Banach*-algebraic properties of $L^{1}(G)$. Suppose that $f \in L^{1}(G)$ is self-adjoint such that $L_{f}: L^{1}(G) \rightarrow L^{1}(G)$ has $(\delta)$ or the weak 2-SDP (for definitions of these and other local spectral properties, see [15]), then [15, Corollary 4.2] implies that 
$\sigma_{L^{1}(G)}(f)=\sigma_{A}(f)$, where $A$ is the completion of $L^{1}(G)$ with respect to an arbitrary $C^{*}$-norm. Hence, if $L_{f}: L^{1}(G) \rightarrow L^{1}(G)$ has the weak 2-SDP for each self-adjoint $f \in L^{1}(G)$, this forces $L^{1}(G)$ to be hermitian and to have a unique $C^{*}$-norm. If $L_{f}: L^{1}(G) \rightarrow L^{1}(G)$ has $(\delta)$ for each self-adjoint $f \in L^{1}(G)$, we even obtain that $L^{1}(G)$ has to be hermitian and *-regular: This follows from [15, Corollary 4.2] and [4, Theorem 2.3], combined with the fact that the quotient of an operator with $(\delta)$ has again $(\delta)$. In particular, whenever $G$ is not hermitian or not amenable, there is a self-adjoint $f \in L^{1}(G)$ such that $L_{f}$ : $L^{1}(G) \rightarrow L^{1}(G)$ is not decomposable.

We thus have a natural, albeit very rough, estimate "from above" for the class of locally compact groups $G$ such that, for each $f \in L^{1}(G)$, the convolution operator $L_{f}$ : $L^{1}(G) \rightarrow L^{1}(G)$ is decomposable. The much harder problem, which is the main topic of this note, is to find an estimate "from below" for this class of groups, going significantly beyond compact and abelian groups.

A locally compact group is called a Moore group if all of its (topologically) irreducible unitary representations are finite-dimensional. All abelian and all compact groups are Moore groups, but the class of Moore groups is much larger. For details, see [18].

For Moore groups, we have the following positive result:

Theorem. Let $G$ be a Moore group, let $E$ a Banach space, and let $\theta: L^{1}(G) \rightarrow \mathscr{B}(E)$ be a continuous homomorphism. Then, for each $f \in L^{1}(G)$, the operator $\theta(f)$ is decomposable.

For the proof of the Theorem, we wish to apply the decomposability criterion [3, Theorem 3.7]. Since [3, Theorem 3.7] is concerned with unital Banach algebras only, whereas we will have to deal with possibly non-unital algebras, we first have to customize the Albrecht-Mehta criterion.

Consider the following situation:

- $\mathfrak{U}$ is a Banach algebra;

- $A$ is a regular Banach subalgebra of the center of $\mathfrak{U}$ and contains an approximate identity for $\mathfrak{U}$;

- if $\mathfrak{U}$ is unital, $A$ contains the identity of $\mathfrak{U}$.

For each $\phi \in \Phi_{A}$, the character space of $\boldsymbol{A}$, let $I_{\phi}$ denote the closed ideal of $\mathcal{U}$ generated by $\operatorname{ker} \phi$. For $a \in \mathfrak{U}$ and $\phi \in \Phi_{A}$, let $\sigma_{\phi}(a)$ denote the spectrum of $a+I_{\phi}$ in $\mathfrak{U} / I_{\phi}$.

Lemma 1. Let the above situation be given, let $E$ be a Banach space, and let $\theta$ : $\mathfrak{U} \rightarrow \mathscr{B}(E)$ be a continuous homomorphism. Let $a \in \mathfrak{U}$ be such that $\sigma_{\phi}(a)$ is totally disconnected for all $\phi \in \Phi_{A}$. Then $\theta(a)$ is decomposable.

Proof. If $\mathfrak{U}$ is unital, and if $\theta(1)=\mathrm{id}_{E}$, the statement is just a special case of [3, 
Theorem 3.7]. Suppose that $\mathfrak{U}$ is unital, but that $p:=\theta(1) \neq \mathrm{id}_{E}$. By [3, Theorem 3.7], we obtain the decomposability of $\theta(a) \mid p E$. Since $\theta(a) \mid\left(\operatorname{id}_{E}-p\right) E \equiv 0$, we see that $\theta(a)$ is in fact decomposable on all of $E$. Finally, let $\mathfrak{U}$ be non-unital. Let $\mathfrak{U}^{\#}$ be the unitization of $\mathfrak{U}$, and let $A^{\#}$ denote the Banach subalgebra of $\mathfrak{U}^{\#}$ generated by $A$ and 1 . It is routinely verified that $A^{\#}$ is regular. Hence, with $\mathfrak{U}^{\#}$ and $A^{\#}$, we are in the same situation as with $\mathfrak{U}$ and $A$. Pick $\phi \in \Phi_{A}$, and let $J_{\phi}$ denote the closed ideal of $\mathfrak{U}^{\#}$ generated by ker $\phi$. If $\phi \mid A \neq 0$, the ideal $J_{\phi}$ equals $I_{\phi}$. Hence, the spectrum of $a+J_{\phi}$ in $\mathfrak{U}^{\#} / I_{\phi}$ equals $\sigma_{\phi}(a)$ and is thus totally disconnected. If $\phi \mid A \equiv 0, J_{\phi}$ equals $\mathfrak{U}$ because $A$ contains an approximate identity for $\mathfrak{U}$. Therefore, the spectrum of $a+J_{\phi}$ is $\{0\}$. Extend $\theta$ to a unital homomorphism $\theta^{\#}: \mathfrak{U}^{\#} \rightarrow \mathscr{B}(E)$. In view of the foregoing, [3, Theorem 3.7] applies and yields the decomposability of $\theta^{\#}(a)=\theta(a)$.

In order to apply Lemma 1 to the situation of the Theorem, we require some notions from non-abelian harmonic analysis (see [9, 10, and 17]).

Let $G$ be a locally compact group, and let $\operatorname{Aut}(G)$ denote the group of topological automorphisms of $G$. Equipped with a natural variant of the compact-open topology ([12, Definition 26.3]), Aut $(G)$ becomes a topological group of its own right. Let $f: G \rightarrow \mathbb{C}$ be a function, and let $\alpha \in \operatorname{Aut}(G)$. We define $f^{\alpha}$ through $f^{\alpha}(x)=f\left(\alpha^{-1}(x)\right)$ for $x \in G$. If $f \in L^{1}(G)$, then $f^{\alpha} \in L^{1}(G)$ as well. For a subset $S$ of $L^{1}(G)$ and $\alpha \in \operatorname{Aut}(G)$, we set $S^{\alpha}:=\left\{f^{\alpha}\right.$ : $f \in S\}$. Let $B$ be a subgroup of $\operatorname{Aut}(G)$ containing $I(G)$, the group of inner automorphisms of $G$. A subset $S$ of $L^{1}(G)$, will be called $B$-invariant if $S^{\alpha} \subset S$ for all $\alpha \in B$. We write $\mathscr{M}_{B}\left(L^{1}(G)\right)$ for the collection of those regular ideals of $L^{1}(G)$ which are maximal among the regular, $B$-invariant ideals of $L^{1}(G)$. In case $B=I(G), \mathscr{M}_{B}\left(L^{1}(G)\right)$ is the space $\mathscr{M}\left(L^{1}(G)\right)$ of maximal regular ideals of $L^{1}(G)$. The $B$-center $Z^{B}\left(L^{1}(G)\right)$ of $L^{1}(G)$ is the closed subalgebra of $L^{1}(G)$ consisting of those $f \in L^{1}(G)$ for which $f^{\alpha}=f$ for all $\alpha \in B$. If $B=I(G)$, then $Z^{B}\left(L^{1}(G)\right)$ is the usual center of $L^{1}(G)$. If $B$ is relatively compact in $\operatorname{Aut}(G)$, we say that $G$ is an $[F I A]_{B}^{-}$-group; in the special case of $B=I(G)$, we suppress the subscript and simply speak of $G$ as of an $[F I A]^{-}$-group. Finally suppose that $G$ is an extension of another locally compact group $N$. Then each $x \in G$ induces an automorphism $\alpha_{x}$ of $N$ via conjugation with $x$. We write $I(N, G)$ for the subgroup $\left\{\alpha_{x}: x \in G\right\}$ of $\operatorname{Aut}(N)$; often, we shall abuse notation and simply write $G$ instead of $I(N, G)$.

Lemma 2. Let $G$ be a finite extension of an $[F I A]^{-}$-group $N$. Then:

(i) $N$ is an $[F I A]_{G}^{-}$-group.

(ii) For each $m \in \mathscr{M}_{G}\left(L^{1}(N)\right)$, there are $\mathrm{m}_{1}, \ldots, \mathrm{m}_{n} \in \mathscr{M}\left(L^{1}(N)\right)$ such that $\mathrm{m}=\mathrm{m}_{1} \cap \ldots \cap$ $\mathbf{m}_{n}$.

Proof. Let $x_{1}, \ldots, x_{n} \in G$ be representatives of the cosets of $N$, and set $\alpha_{j}:=\alpha_{x_{j}}$ for $j \in\{1, \ldots, n\}$. Since $I(N)$ is relatively compact in $\operatorname{Aut}(N)$, the same is true for each of its cosets. For each $x \in G$, there is $j \in\{1, \ldots, n\}$ and $y \in N$ such that $x=x_{j} y$ and thus $\alpha_{x}=\alpha_{j} \alpha_{y}$. Hence, we have $I(N, G)=\bigcup_{j=1}^{n} \alpha_{j} I(N)$, and in particular, $I(N, G)$ is relatively compact in $\operatorname{Aut}(N)$. This proves (i).

For the proof of (ii), let $m \in \mathscr{M}_{G}\left(L^{1}(N)\right)$. In particular, $m$ is a regular ideal of $L^{1}(G)$. Hence, there is a maximal regular ideal $M$ of $L^{1}(N)$ such that $m \subset M$. For $j=1, \ldots, n$, set 
$m_{j}:=M^{a_{j}}$. Since $N$ is a normal subgroup of $G, m_{j}$ is an ideal of $L^{1}(N)$ for $j=1, \ldots, n$. If $u \in L^{1}(N)$ is a modular unit for $M$, then it is a routine matter to verify that $u^{\alpha_{j}}$ is a modular unit for $\mathrm{m}_{j}$ for $j=1, \ldots, n$. Further, if for some $j \in\{1, \ldots, n\}$ there is a proper, regular ideal $\tilde{M}$ containing $\mathrm{m}_{j}$, then $\tilde{M}^{\alpha_{j}^{-1}}$ is easily seen to be a regular ideal containing $M$. Since $M$ was assumed to be maximal, we see that $\tilde{M}^{\alpha j^{-1}}=M$ and therefore $\tilde{M}=\mathrm{m}_{j}$. Consequently, $\mathrm{m}_{1}, \ldots, \mathrm{m}_{n}$, belong to $\mathscr{M}\left(L^{1}(N)\right)$. Since $\mathrm{m}$ is $G$-invariant, we have

$$
\mathbf{m}=\mathbf{m}^{\alpha_{j}} \subset M^{\alpha_{j}}=\mathbf{m}_{j} \quad(j=1, \ldots, n),
$$

i.e. $m \subset m_{1} \cap \ldots \cap m_{n}$. We now claim that $m_{1} \cap \ldots \cap m_{n}$ is also $G$-invariant. Fix $f \in \mathrm{m}_{1} \cap \ldots \cap \mathrm{m}_{n}$ and $x \in G$. By definition of $\mathrm{m}_{1} \ldots, \mathrm{m}_{n}$, there is, for each $j \in\{1, \ldots, n\}$, an element $g_{j} \in M$ such that $f=g_{j}^{\alpha_{j}}$. Moreover, for each $j \in\{1, \ldots, n\}$, there are $k(j) \in\{1, \ldots, n\}$ and $y \in N$ such that $x x_{j}=x_{k(j)} y$. Thus, we have

$$
f^{\alpha_{j}}=\left(g_{j}^{\alpha_{j}}\right)^{\alpha_{x}}=g_{j}^{\alpha_{x} \alpha_{j}}=f_{j}^{\alpha_{k(j) \alpha_{y}}}=\left(f_{j}^{\alpha_{y}}\right)^{\alpha_{k(j)}} \in \mathfrak{m}_{k(j)} \quad(j \in\{1, \ldots, n\}) .
$$

It is easy to see that the assignment $\{1, \ldots, n\} \ni j \rightarrow k(j)$ is a bijection. Hence, we obtain $f^{\alpha_{x}} \in \mathfrak{m}_{1} \cap \ldots \cap \mathfrak{m}_{n}$. Since $m$ is a maximal regular, $G$-invariant ideal of $L^{1}(G)$, we conclude that $m=m_{1} \cap \ldots \cap m_{n}$.

Proof of the Theorem. By [20, Theorem 1], $G$ is a finite extension of a Takahashi group $N$ (for the definition of Takahashi groups, see [18]). Since $N$ is open in $G$, we can identify $L^{1}(N)$ with the subalgebra of $L^{1}(G)$ consisting of those $f \in L^{1}(G)$ whose support lies in $N$. It is well known $([18, p .701])$ that every Takahashi group is an $[F I A]^{-}$-group. From Lemma 2(i), we conclude that $N$ is also an $[F I A]_{G}^{-}$-group. By $[16$, Proposition 2.4], this implies that $Z^{G}\left(L^{1}(N)\right)$ is a regular commutative Banach algebra. Since Moore groups are [SIN]-groups, and since $N$ is open, we see that $Z^{G}\left(L^{1}(N)\right)$ contains a bounded approximate identity of for $L^{1}(G)$. Hence-with $\mathfrak{U}=L^{1}(G)$ and

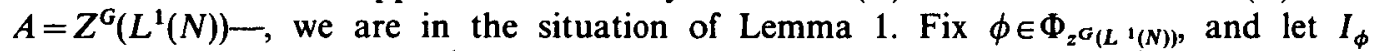
denote the closed ideal of $L^{1}(G)$ generated by $\operatorname{ker} \phi$. We claim that $\operatorname{dim} L^{i}(G) / I_{\phi}<\infty$. By [21, Lemma 3.1], it suffices to show that $L^{1}(N) \cap I_{\phi}$ has finite codimension in $L^{1}(N)$. Let $J_{\phi}$ be the closed ideal of $L^{1}(N)$ generated by ker $\phi$. Clearly, $J_{\phi} \subset L^{1}(N) \cap I_{\phi}$. Using [17, Theorem 4.5], we see that the hull of $J_{\phi}$ in $\mathscr{M}_{G}\left(L^{1}(N)\right)$ consists of exactly one ideal. Thus, by Lemma 2(ii), the hull of $J_{\phi}$ in $\mathscr{M}\left(L^{1}(N)\right)$ is finite. Since $N$ is also a Moore group, we conclude from [11] that $\operatorname{dim} L^{1}(N) / J_{\phi}<\infty$ and thus $\operatorname{dim} L^{1}(G) / I_{\phi}<\infty$. An application of Lemma 1 completes the proof.

Corollary. Let $G$ be a Moore group, and let $f \in L^{1}(G)$. Then the convolution operator $L_{f}: E \rightarrow E$ is decomposable whenever $E$ is any of the following: $\mathscr{C}_{0}(G), M(G), L^{p}(G)$ for $p \in[1, \infty]$, or $C^{*}(G)$.

Remarks. 1. The strength of the Theorem lies in the fact that it ascertains the decomposability of $\theta(f)$ for all $f \in L^{1}(G)$. Older results establish the decomposability of 
$\theta(f)$ for certain $f$. For instance, if $G$ has polynomial growth, and if $f \in L^{1}(G) \cap L^{2}(G)$ is self-adjoint with compact support, then $\theta(f)$ is decomposable: This follows from [6] and [2]. In fact, if $G$ is a compactly generated [SIN]-group with polynomial growth, we even have decomposability of $\theta(f)$ for all self-adjoint $f$ belonging to a certain Beurling subalgeba of $L^{1}(G)$ : This is a consequence of [22, Proposition 2.2(ii)]. In both cases, $\theta$ need not be continuous.

2. Local spectral theory has substantial applications in automatic continuity ([13]). In this context, it would be interesting to know if the continuity assumption for $\theta$ in the Theorem can be dropped. The problem here is that the proof of [3, Theorem 3.7] crucially relies on [3, Lemma 3.5] for whose proof the continuity of $\theta$ seems to be indispensable.

3. Let $G$ be a locally compact group, and let $H$ be a closed subgroup of $G$. Then we may view $M(H)$, and hence $L^{1}(H)$, as a subalgebra of $M(G)$. Since a closed subgroup of a Moore group is again a Moore group, we have the following strengthening of the Corollary:

Let $G$ be a Moore group, let $H$ be a closed subgroup of $G$, and let $\mu \in L^{1}(H)$. Then the convolution operator $L_{\mu}: E \rightarrow E$ is decomposable whenever $E$ is any of the following: $\mathscr{C}_{0}(G), M(G), L^{P}(G)$ for $p \in[1, \infty]$, or $C^{*}(G)$.

The last remark shows that if $G$ is a Moore group, we may find $\mu \in M(G) \backslash L^{1}(G)$ such that nevertheless $L_{\mu}: L^{1}(G) \rightarrow L^{1}(G)$ is decomposable. In case $G$ is LCA, $L_{\mu}: L^{1}(G) \rightarrow L^{1}(G)$ is also decomposable whenever $\mu$ is discrete. Concluding this note we give an example of a discrete measure $\mu$ on a compact group $G$ such that $L_{\mu}: L^{1}(G) \rightarrow L^{1}(G)$ lacks almost every important local spectral property.

Example. Let $G$ be a compact group which contains $\mathbb{F}_{2}$, the free group on two generators, as a subgroup. For instance, let $G:=S U(2)$, the group of all unitary $2 \times 2$ matrices with determinant 1 ([19, Proposition 3.2]). Fix two elements $a, b \in G$ which generate $\mathbb{F}_{2}$, and let $\mu$ be the discrete probability measure on $G$ which is concentrated on $\left\{a, b, a^{-1}, b^{-1}\right\}$ such that

$$
\mu(\{a\})=\mu(\{b\})=\mu\left(\left\{a^{-1}\right\}\right)=\mu\left(\left\{b^{-1}\right\}\right) .
$$

We may view $\mu$ as an element of $\ell^{1}\left(\mathbb{F}_{2}\right)$. In [8, Chapter 3], the spectrum of $\mu$ in $\ell^{1}\left(\mathbb{F}_{2}\right)$ is computed:

$$
\sigma_{\ell^{1}\left(\mathbb{F}_{2},\right.}(\mu)=\left\{z=x+i y \in \mathbb{C}: x^{2}+2 y^{2} \leq 1\right\},
$$

i.e. $\sigma\left(L_{\mu} \mid L^{1}(G)\right)=\sigma_{M(G)}(\mu)=\sigma_{\ell^{1}\left(F_{2}\right)}(\mu)$ is a non-degenerate ellipse. Consider the inclusion $L^{2}(G) \subset L^{1}(G)$ : It has dense range and intertwines $L_{\mu}: L^{2}(G) \rightarrow L^{2}(G)$ and $L_{\mu}: L^{1}(G) \rightarrow$ $L^{1}(G)$. Since $L_{\mu}: L^{2}(G) \rightarrow L^{2}(G)$ is self-adjoint, we have $\sigma\left(L_{\mu} \mid L^{2}(G)\right) \subset \mathbb{R}$ and, in particular, $\sigma\left(L_{\mu} \mid L^{1}(G)\right) \notin \sigma\left(L_{\mu} \mid L^{2}(G)\right)$. From [15, Theorem 4.2(a)], we conclude that $L_{\mu}: L^{1}(G) \rightarrow$ $L^{1}(G)$ cannot have $(C)$. Further, $\mu$ is canonically contained in $M\left(C^{*}(G)\right.$ ), the multiplier algebra of $C^{*}(G)$, i.e. we may speak of $L_{\mu}: C^{*}(G) \rightarrow C^{*}(G)$. Since $\mu$ as an element of 
$M\left(C^{*}(G)\right)$ is self-adjoint, we have $\sigma\left(L_{\mu} \mid C^{*}(G)\right) \subset \mathbb{R}$ and hence $\sigma\left(L_{\mu} \mid L^{1}(G)\right) \notin \sigma\left(L_{\mu} \mid C^{*}(G)\right)$. Since the inclusion $L^{1}(G) \hookrightarrow C^{*}(G)$ intertwines $L_{\mu}: L^{1}(G) \rightarrow L^{1}(G)$ and $L_{\mu}: C^{*}(G) \rightarrow C^{*}(G)$, $\left[15\right.$, Theorem 4.1(b) and (c)] imply that $L_{\mu}: L^{1}(G) \rightarrow L^{1}(G)$ has neither $(\delta)$ nor the weak 2-SDP. However, $L_{\mu}: L^{1}(G) \rightarrow L^{1}(G)$ does have the SVEP: This follows easily from the fact that the finite-dimensional *-representations of $M(G)$ separate its points.

\section{REFERENCES}

1. C. A. Aremann, Some mapping properties of the group algebras of a compact group, Pacific J. Math. 22 (1967), 1-8.

2. E. Albrecht, Decomposable systems of operators in harmonic analysis, in Toeplitz Centennial (I. GohberG, ed, Birkhäuser, 1982), pp. 13-37.

3. E. Albrecht and R. D. Menta, Some remarks on local spectral theory, J. Operator Theory 12 (1984), 285-317.

4. B. A. Barnes, The properties *-regularity and uniqueness of $C^{*}$-norm in a general *-algebra, Trans. Amer. Math. Soc. 279 (1983), 841-859.

5. I. ColojoarA and C. Folaş, Theory of Generalized Spectral Operators (Gordon and Breach, 1968).

6. J. Dixmier, Opérateurs de rang fini dans les représentations unitaires, Publ. Math. I.H.E.S. 6 (1960), 13-25.

7. J. EsChmeIER, Operator decomposability and weakly continuous representations of locally compact abelian groups, J. Operator Theory 7 (1982), 201-208.

8. A. Figa-Talamanca and M. A. Picardello, Harmonic Analysis on Free Groups (Marcel Dekker, 1983).

9. S. Grosser and M. Moskowitz, On central topological groups, Trans. Amer. Math. Soc. 127 (1967), 317-340.

10. S. Grosser and M. Moskowitz, Compactness conditions in topological groups, J. Reine Angew. Math. 246 (1971), 1-40.

11. W. Hauenschild and E. Kaniuth, The generalized Wiener theorem for groups with finite-dimensional representations, J. Funct. Anal. 31 (1979), 13-23.

12. E. Hewitt and K. A. Ross, Abstract Harmonic Analysis, I (Springer Verlag, 1963).

13. K. B. Laursen and M. M. Neumann, Decomposable operators and automatic continuity, $J$. Operator Theory 15 (1986), 33-51.

14. K. B. Laursen and M. M. Neumann, Decomposable multipliers and applications to harmonic analysis, Studia Math. 101 (1992), 193-214.

15. K. B. Laursen and M. M. Neumann, Asymptotic intertwining and spectral inclusions on Banach spaces, Czechoslovak Math. J. 43 (1993), 483-497.

16. J. LiukKonen and R. D. Mosak, Harmonic analysis and centers of group algebras, Trans. Amer. Math. Soc. 195 (1974), 147-163.

17. R. D. Mosak, The $L^{1}$ - and $C^{*}$-algebras of $[F A I]_{B}^{-}$-groups and their representations, Trans. Amer. Math. Soc. 163 (1972), 277-310.

18. T. W. PaLmer, Classes of nonabelian, noncompact, locally compact groups, Rocky Mountain J. Math. 8 (1978), 681-741. 
19. A. T. Paterson, Amenability (American Mathematical Society, 1988).

20. L. C. Robertson, A note on the structure of Moore groups, Bull. Amer. Math. Soc. 75 (1969), 594-599.

21. V. Runde, Homomorphisms from $L^{1}(G)$ for $G \in[F I A]^{-} \cup$ [Moore], J. Funct. Anal. 122 (1994), 25-51.

22. V. RUNDE, Intertwining operators over $L^{1}(G)$ for $G \in[P G] \cap[S I N]$, Math. Z., to appear.

Fachbereich 9 MathematiK

UNIVERSITÄT DES SAARLANDES

POSTFACH 151150

66041 SAARBRÖCKEN

Germany

E-mail address: runde@math.uni-sb.de 